Preprint typeset in JINST style - HYPER VERSION

\title{
Hadron beam test of a scintillating fibre tracker system for elastic scattering and luminosity measurement in ATLAS
}

\author{
F. Anghinolfi ${ }^{a}$, S. Ask ${ }^{a}$, P. Barrillon ${ }^{b}$, G. Blanchot ${ }^{a}$, S. Blin ${ }^{b}$, A. Braem ${ }^{a}$, C. de La \\ Taille $^{b}$, B. Di Girolamo ${ }^{a}$, I. Efthymiopoulos ${ }^{a}$, J. Faustino $^{c, e}$, D. Fournier ${ }^{b}$, S. Franz $^{a}$, \\ P. Grafström ${ }^{a}$, L. Gurriana ${ }^{e}$, M. Haguenauer ${ }^{f}$, V. Hedberg $^{g}$, M. Heller $^{b}$, S. Hoffmann ${ }^{h}$, \\ W. Iwanski ${ }^{a, i}$, C. Joram ${ }^{a}$, A. Kočnár ${ }^{j}$, B. Lavigne ${ }^{b}$, B. Lundberg ${ }^{g}$, A. Maio ${ }^{d, e}$, \\ M.J.P. Maneira ${ }^{c}$, A. Mapelli ${ }^{a}$, C. Marques $^{e}$, U. Mjörnmark $^{g}$, P. Conde Muíño ${ }^{e}$, \\ P. Puzo $^{b}$, M. Rijssenbeek ${ }^{k}$, J.G. Saraiva ${ }^{d, e}$, N. Seguin-Moreau ${ }^{b}$, S. Soares ${ }^{d, e}$, \\ H. Stenzel ${ }^{h}$, M. Thioye ${ }^{k}$, D. Varouchas ${ }^{b}$ and V. Vorobel $^{j}$ \\ ${ }^{a}$ CERN, PH Department, Geneva, Switzerland, \\ ${ }^{b}$ Laboratoire de l'Accelerateur Lineaire, Orsay, France \\ ${ }^{c}$ CEFITEC/Faculdade de Ciências e Tecnlologia da Universidade Nova de Lisboa, Lisbon, \\ Portugal \\ ${ }^{d}$ CFNUL/Faculdade de Ciências da Universidade de Lisboa, Lisbon, Portugal \\ ${ }^{e}$ LIP - Laboratório de Fisíca Experimental e Instrumenta cão em Partículas, Lisbon, Portugal \\ ${ }^{f}$ Ecole Polytechnique, Palaiseau, France \\ ${ }^{g}$ University of Lund, Lund, Sweden \\ ${ }^{h}$ II. Physikalisches Institut, Justus-Liebig-Universität, Giessen, Germany \\ iFJ PAN, Krakow, Poland \\ ${ }^{j}$ Faculty of Mathematics and Physics, Charles University in Prague, Czech Republic \\ ${ }^{k}$ Stony Brook University, New York, USA \\ E-mail: Stefan.Askecern.ch
}

ABSTRACT: A scintillating fibre tracker is proposed to measure elastic proton scattering at very small angles in the ATLAS experiment at CERN. The tracker will be located in so-called Roman Pot units at a distance of $240 \mathrm{~m}$ on each side of the ATLAS interaction point. An initial validation of the design choices was achieved in a beam test at DESY in a relatively low energy electron beam and using slow off-the-shelf electronics. Here we report on the results from a second beam test experiment carried out at CERN, where new detector prototypes were tested in a high energy hadron beam, using the first version of the custom designed front-end electronics. The results show an adequate tracking performance under conditions which are similar to the situation at the LHC. In addition, the alignment method using so-called overlap detectors was studied and shown to have the expected precision.

KEYWORDS: Particle tracking detectors; Scintillators and scintillating fibres and light guides.

\footnotetext{
${ }^{*}$ Corresponding author.
} 


\section{Contents}

1. Introduction 1

2. The CERN testbeam setup 2

2.1 Prototype fibre detectors

2.2 The ALFA electronics and system setup

3. The data analysis

3.1 The ALFA data quality 5

3.2 Space point reconstruction

3.2.1 Stand-alone method 6

3.2.2 ALFA versus Si-telescope

3.3 The overlap detectors 8

4. Conclusions 9

5. Acknowledgments 10

\section{Introduction}

New physics at the LHC might manifest itself as deviations from the Standard Model predictions of cross sections and thus the normalization of the measured cross sections is of utmost importance. The uncertainty of the absolute luminosity is the main factor limiting the measurements precision. Traditionally, the absolute luminosity at hadron colliders was determined via elastic scattering of protons at small angles. This is also one of the approaches pursued by the ATLAS experiment at the LHC. ATLAS is aiming to measure at such small angles that the elastic scattering becomes sensitive to the well-known electro-magnetic amplitude. A scintillating fibre tracker system, called ALFA, is used to measure the scattered protons. Fibre tracker modules will be inserted in so-called Roman Pot units which makes it possible to detect scattered protons very close to the beam. The vertically arranged Roman Pots penetrate into the LHC beam pipe on each side of the ATLAS experiment at a distance of $240 \mathrm{~m}$ from the interaction point. The luminosity measurement and the requirements on the detector have been described in [1]. The main requirements which are directly related to the fibre tracker design, such as light yield, sensitivity at the fibre edge etc., were shown to be fulfilled in a beam test of ALFA at DESY in November 2005 [2].

A major difference between the 2005 setup and the final system is the dedicated ALFA frontend (FE) electronics which will be used in ATLAS. The previously used analog readout that was based on standard (NIM and VME) electronics does not meet requirements at the LHC, such as a $40 \mathrm{MHz}$ clock frequency and intermediate data storage during the trigger latency time. For this 
reason, dedicated FE electronics have been designed for ALFA. The new electronics are entirely based on binary hit information. The number of channels, the available space and the remote location of the final detectors are challenges for the design of the FE electronics. To operate ALFA with the final components is therefore a crucial validation step of the overall system design.

The measured spatial resolution in the previous test of ALFA was shown to be limited by multiple scattering of the relatively low energy, $6 \mathrm{GeV} / \mathrm{c}$, electron beam. This also motivated a second test of the fibre tracker in a high energy hadron beam, which approaches the situation in the LHC and where effects due to multiple scattering are negligible. The results from the DESY test together with GEANT4 simulations made it possible to predict that the obtained spatial resolution of $36 \mu \mathrm{m}$ would decrease to about $20-25 \mu \mathrm{m}$ if ALFA was operated in a high energy hadron beam.

The precise relative vertical distance of the upper and lower ALFA detectors in one Roman Pot unit cannot be derived from the physics data, but will be measured by the so-called Overlap Detectors (OD), also based on scintillating fibre technology. The ODs need to measure only the vertical coordinate. In the final ALFA setup the ODs will be located in special extrusions of the Roman Pots which will gradually overlap when the upper and lower pots are moved towards the beam axis. These detectors, as well as the alignment method, were tested for the first time in this experiment.

\section{The CERN testbeam setup}

The beam test was carried out at the CERN SPS H8 beam which is a secondary beam in the CERN North Area. The primary target consists of a $30 \mathrm{~cm}$ long Beryllium plate. We have used for most of the period a secondary beam at $230 \mathrm{GeV} / \mathrm{c}\left(70 \% p\right.$ and $\left.30 \% \pi^{+}\right)$and for a few days a $20 \mathrm{GeV} / \mathrm{c}$ secondary beam (mainly $\pi^{+}$beam with a small $p$ contamination). The beam spot size was between $1-10 \mathrm{~mm}$, and varied with the beam line settings, and the beam divergence was about $0.15 \mathrm{mrad}$.

The ALFA detector was mounted on a table which allowed the detector to be translated in the direction of the axes of the plane transverse to the beam, as well as being rotated around these axes. The trigger was based on a $30 \times 30 \mathrm{~mm}^{2}$ plastic scintillator and its signal was put in coincidence with the signal from a second scintillator that had dimensions similar to the active area of the particular ALFA prototype used.

\subsection{Prototype fibre detectors}

Two new ALFA prototypes [4, 5] were built with an increased number of fibres compared to the DESY testbeam, however, their design and construction followed the same principles. A detector plane consists of a ceramic substrate which on both sides supports Aluminium coated square fibres ${ }^{1}$ of $500 \mu \mathrm{m}$ thickness in a so-called UV configuration, i.e. the two fibre layers have an angle of $\pm 45^{\circ}$ relative to the vertical axis as shown in the left part of figure 1. In the final detector, ten such UV planes with two times 64 fibres will be assembled with a relative staggering of multiples of $\sqrt{2} \cdot 50 \mu \mathrm{m}$.

The first detector constructed for the testbeam, called ALFA 2_2_64, included two planes which contained the full set of 64 fibres per layer. It was tested with a prototype of the final

\footnotetext{
${ }^{1}$ Fibre type SCSF-78, single cladded, S-type, from Kuraray, Japan
} 


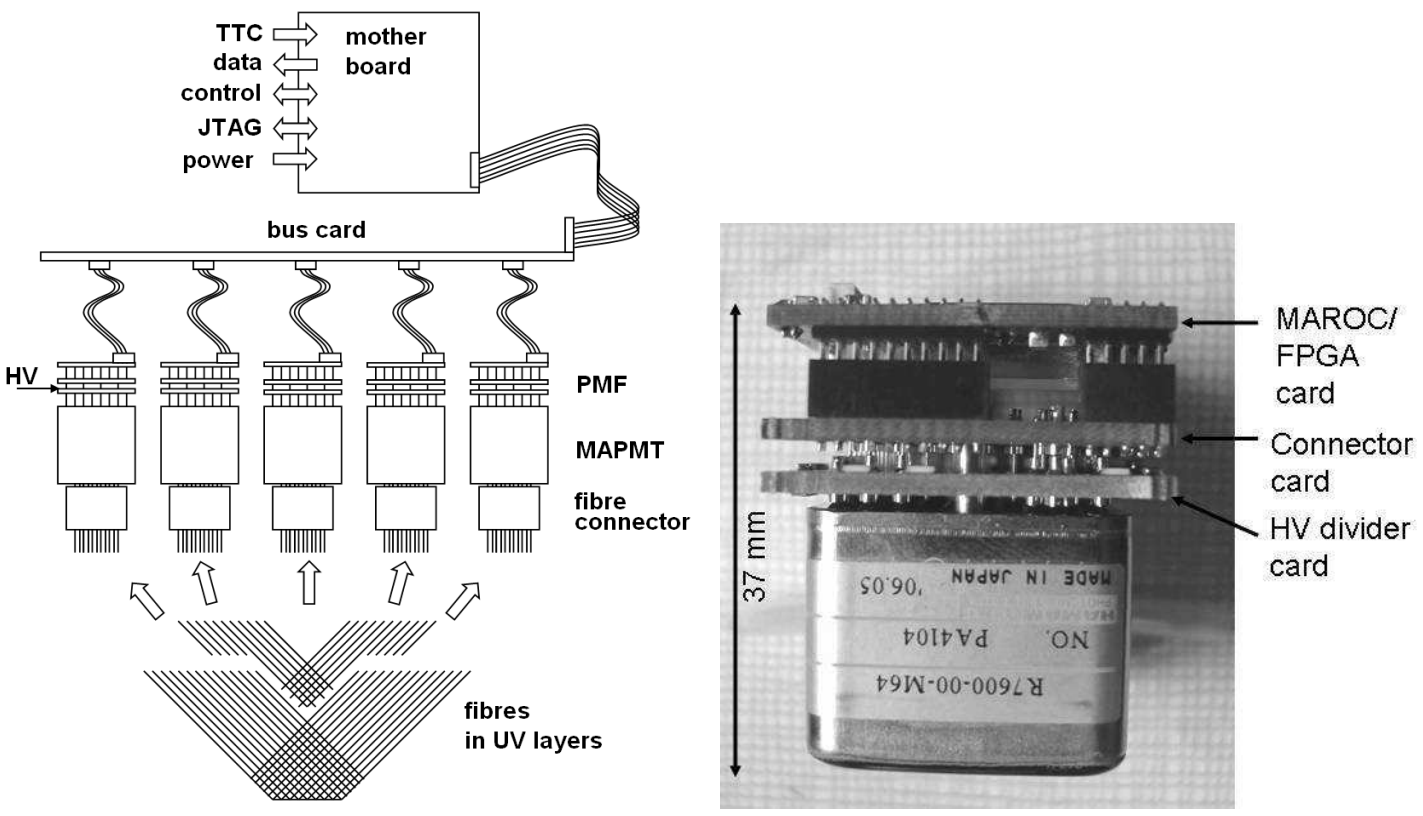

Figure 1. Schematic representation of the testbeam system setup (left). The MAPMT together with the three PCBs making a PMF unit (right).

trigger scintillator whose shape matches the overlap area of the fibres. It was connected via a specially formed light guide to a $\mathrm{PMT}^{2}$. The challenges with this trigger counter is the stringent space limitations in the pot and the requirement of a very uniform trigger efficiency in order not to bias the measurement. Lab tests with a Sr-90 source showed a photoelectric yield in excess of 30. Setting the trigger threshold at $\approx 5$ p.e. should guarantee an excellent uniformity. The second ALFA tracker comprised of 10 staggered planes with 16 fibres per layer (ALFA 10_2_16) and was used for resolution studies.

In addition to the two ALFA trackers, two prototype Overlap Detectors were built [6]. They consist of horizontally mounted scintillating fibres of the same type and size as the main detector. The fibres are organized in two staggered layers with 30 fibres each. The two detectors were placed in the beam such that they overlapped vertically. Their relative vertical position could be mechanically controlled by a micrometric screw with a precision of a few microns.

The scintillating fibres were routed over typically $25 \mathrm{~cm}$ to custom designed fibre connectors with 64 channels in a $8 \times 8$ configuration, which matches precisely the segmentation of the multi anode PMTs ${ }^{3}$. The connectors fit in slots of the Roman Pot vacuum flange, on the top (air) side of which are mounted the MAPMTs with their front-end electronics.

All detectors underwent careful metrological analysis with a 3D coordinate measurement machine. The positions and slopes of all fibres were individually determined and stored in a database. These parameters were then used during the offline-analysis for the space point reconstruction.

\footnotetext{
${ }^{2}$ Hamamatsu H3164. Active diameter $8 \mathrm{~mm}$.

${ }^{3}$ MAPMT type R7600, Hamamatsu, Japan
} 


\subsection{The ALFA electronics and system setup}

A very compact design was adopted for the dedicated ALFA FE electronics, where a large part of the electronics is directly mounted onto the MAPMTs. The right part of figure 1 shows the so-called PMF (PhotoMultiplier Front-end) unit, consisting of three PCBs mounted on top of the MAPMT. From the bottom, the first board is a high voltage (HV) divider which is followed by a board which routes the relevant signals from the MAPMT to the third board. The third board contains the multi anode readout chip (MAROC) [7], providing amplifiers and discriminators for each of the 64 MAPMT channels, and a FPGA for handling the readout.

The readout of the detector follows the ATLAS requirements [8, 9] and is controlled by a motherboard. When the trigger signal arrives to the ALFA detector it is received and fanned-out by the motherboard to the different PMFs. Each PMF stores the data from the MAPMT at $40 \mathrm{MHz}$ in a pipeline and at the arrival of a trigger it sends back the data from the corresponding pipeline location. Due to the synchronous trigger and readout system, the latency of the trigger has to be timed in carefully with respect to the data in the pipeline. The data from all the PMFs is then collected by the motherboard where it is formated and sent through a gigabit optical link (GOL) [10] to the readout system. The configuration of the FE components is done using an embedded local monitor board (ELMB) [9] which is part of the motherboard.

The first version of the ALFA FE electronics was used during the testbeam period and the setup consisted of 5 PMFs, distributed in a row (as is foreseen for the final detector), and a motherboard. The left part of figure 1 shows a schematic view of the setup, with the motherboard at the top and the cabling structure to distribute signals to the 5 PMFs. It should be noted that the interconnection between the motherboard and the PMFs will be based on light Kapton cables in the final system, to replace the large flat cables and adapters used at the testbeam.

A computer with PVSS was used to configure the electronics installation in the testbeam area, through a CAN BUS and the ELMB. The trigger signal was sent through an optical fibre to the motherboard by standard components developed for the ATLAS timing, trigger and control (TTC) [8] system. The trigger-busy logic was made using standard NIM electronics and the DAQ software was implemented within the standard ATLAS frame work. A readout PC was equipped with a FILAR card [11] to read data through the GOL that was connected to the motherboard. The event rate was restricted by the ad hoc implementation of the readout system where a peak rate of about $1.5 \mathrm{kHz}$ and an average rate of about $250 \mathrm{~Hz}$ were obtained.

In the CERN testbeam all the components of the final system were used except for the socalled ROD (ReadOut Driver) card which forms the interface between the GOLs and the common ATLAS readout system. During the tests of the electronics several imperfections were identified. These were strongly suspected of creating fake noise hits (as discussed below) and will be corrected in the next version of the electronics.

\section{The data analysis}

After converting the raw data into an appropriate format, the analysis was entirely based on the ROOT analysis software [12]. A set of basic analysis routines were used during the testbeam to monitor the data during the data-taking, however, the main analysis was carried out after the testbeam period. 
After an initial running period, where the trigger and readout system was timed in and the basic detector operation was established, the beam time was split into one period for each detector prototype. Between 2-7 million events were recorded with each detector and the high energy proton beam was used for the runs dedicated to resolution studies. In addition, some 100k events were recorded together with the setup of the DEPFET collaboration who were starting tests after the ALFA period with a high precision Si-telescope in the same zone.

\subsection{The ALFA data quality}

The overall quality of the data taken during the beam period was fully satisfactory. No problems due to bad synchronization or data corruption were encountered. However, as mentioned before, there were several imperfections in the electronics that were suspected of creating fake noise hits. In order to disentangle the possible different noise sources (fibres, fibre-PMT interface, electronics) we exploited different experimental configurations:

A) A complete MAPMT or individual MAPMT channels, that were not connected to fibres (e.g. in ALFA 2_2_64 or OD);

B) MAPMTs that were connected to, OD, fibres that were not in the beam;

C) MAPMTs that were connected to fibres that were in the beam.

The observed probability of registering a hit was calculated as the number of measured hits in the whole MAPMT divided by the number of channels (64) and the number of events. For our standard HV and threshold setting, the overall noise level for MAPMTs without any fibres connected (case A) was of the order of $0.02 \%$, increasing to $0.4 \%$ for MAPMTs that were connected to fibres, when the fibres were not in the beam (case B).

The noise level also showed correlations with the number of hits registered in the MAPMT which hints at some coupling in the read out chain (analogue or digital).

For MAPMTs which read out fibres that were in the beam (case $\mathrm{C}$ ), the noise was studied by the occurrence of neighbours of a selected hit. For a given hit, the probability of registering a hit in a direct neighbouring MAPMT channel was of the order of $10 \%$. This level decreased to about 5\% when only hits associated to reconstructed tracks were considered. This selection eliminates randomly distributed noise hits which were typically produced in groups with several hits at the same time. The existence of correlated noise was also supported by the analysis of the hit multiplicity per fibre layer. Ideally it should be one (assuming $100 \%$ efficiency, zero cross-talk and no noise), however the measured hit multiplicity showed a mean of two with tails of several tens of fibres hits.

\subsection{Space point reconstruction}

One of the main goals of this test experiment was to measure the intrinsic spatial resolution of the detector. As mentioned earlier the value found at the DESY testbeam $\left(\sigma_{x}=\sigma_{y}=36 \mu \mathrm{m}\right)$, was limited mainly by multiple scattering in the relatively low energy electron beam and should significantly improve in a high energy hadron beam.

All studies of the tracking performance were based on the data taken with the ALFA 10_2_16 detector. The problems with the data quality, discussed above, called for an improved tracking 

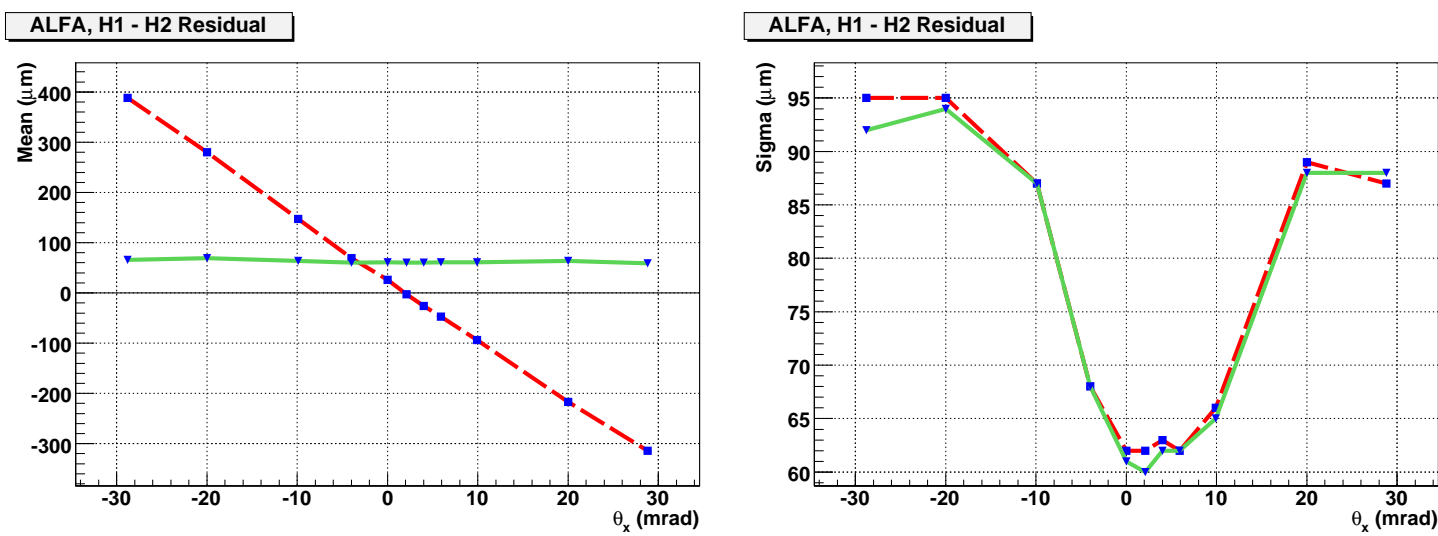

Figure 2. The mean and sigma of the $x_{H 1}-x_{H 2}$ distribution as a function of the $\theta_{x}$ angle. The solid line is based on the reconstructed $x$ coordinates and the dashed line on the $y$ coordinates.

algorithm, which was largely immune to fake noise hits. In a first stage, hit candidates were selected based on a seed track obtained by a Hough transform method [14]. Here the closest hit to the seed track in each ALFA layer was selected under the condition that it was within a distance of 2 fibre widths from the seed track. The selected hits were then used by a so-called minimum overlap algorithm [2] in order to reconstruct the impact point in the $x-y$ plane transverse to the beam. This algorithm assumes that the tracks are perpendicular to the ALFA layers and the $z$ coordinate information therefore becomes irrelevant.

\subsubsection{Stand-alone method}

In the first part of the resolution study, tracks were reconstructed by ALFA as two independent track segments, using planes 1-5 (layers 1-10 = H1) and planes 6-10 (layers 11-20 = H2) separately. In this way the ALFA resolution can be studied without an external tracking detector, however, with limited possibilities to estimate the efficiency. In the following we call this method the stand-alone method. For this study, events were only used if their $x-y$ position was within a $2 \mathrm{~mm}$ radius from the centre of ALFA which ensured that the selected particles traversed a region of ALFA where all 20 layers physically overlap. All following studies were done for both the $x$ and $y$ coordinates, but for simplicity only one will be discussed if the results for the other coordinate were the same.

Before taking data dedicated for resolution studies, an angular scan was made in order to align ALFA with respect to the beam axis. Runs were taken with ALFA rotated around both the $x$ and $y$ axis and during these runs the MAPMTs were operated at $900 \mathrm{~V}$. The runs were analyzed based on the mean value and sigma of the $x_{H 1}-x_{H 2}$ track residual distributions. Figure 2 shows the mean (left) and sigma (right) of the residual distribution as functions of the rotation angle around the $x$-axis, $\theta_{x}$, with respect to the original position of ALFA.

Since the minimum overlap algorithm is reconstructing the $x$ and $y$ coordinate of the particle separately for the two halves, the presence of an angle introduces a systematic shift of the mean of the distribution. This is clearly seen in figure 2 where the mean in $y$ shifts linearly with the $\theta_{x}$ angle and where a perfectly perpendicular alignment of ALFA to the beam corresponds to a mean equal to zero. The shift of the mean therefore provides a method for a precise alignment $(\sim 1 \mathrm{mrad})$, 


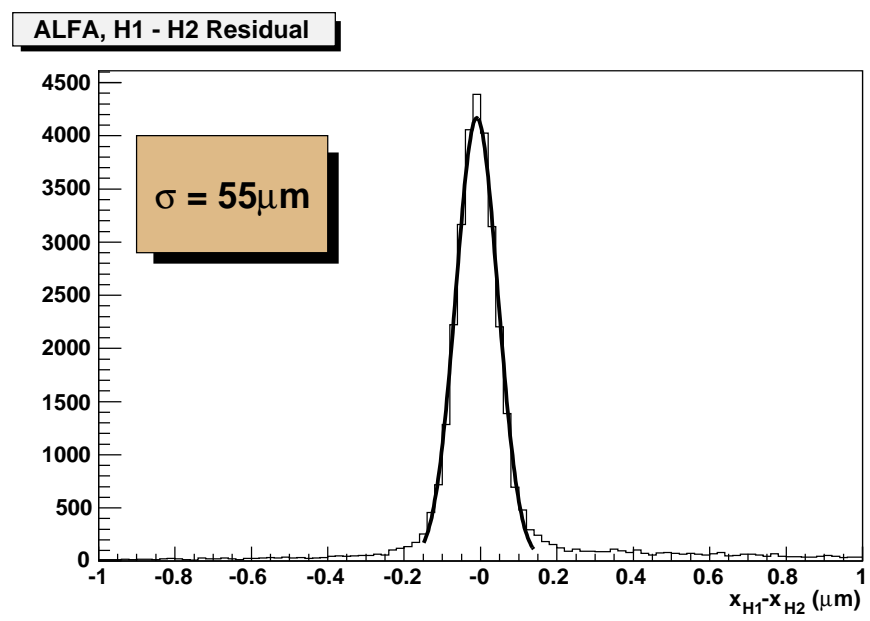

Figure 3. The residual distribution from the $x$-coordinates reconstructed by the two ALFA halves, $H 1$ and $H 2$.

which will also be useful when operating at the LHC. Figure 2 also shows the residual sigma as a function of $\theta_{x}$ where the minimum is consistent with an optimal alignment of about $\theta_{x}=2 \mathrm{mrad}$ obtained from the results based on the mean of the distribution.

After the alignment, dedicated high statistics runs were taken in order to study the spatial resolution of ALFA. During these runs ALFA was operated both at $900 \mathrm{~V}$ and $950 \mathrm{~V}$. Figure 3 shows the residual distribution obtained from high statistics runs taken at $950 \mathrm{~V}$. A Gaussian fit to the distribution gives $\sigma=55 \pm 0.3 \mu \mathrm{m}$ (only statistical error from the fit) and as both half detectors are practically identical, the spatial resolution of each of them is expected to be $\sigma_{H 1}=\sigma_{H 2}=$ $55 / \sqrt{2} \sim 39 \mu \mathrm{m}$.

Comparison with the output from a geometrical Monte-Carlo (MC) [4], with the as-measured detector geometry as input, allows us to interpret the measured value as a spatial resolution of the full ALFA detector,

$$
\sigma_{A L F A}=\frac{\sigma_{H} \ominus \sigma_{g e o}}{2} \oplus \sigma_{g e o}=\frac{\sqrt{\sigma_{H}^{2}+3 \cdot \sigma_{g e o}^{2}}}{2}
$$

where $\sigma_{H}$ is the measured half ALFA resolution and $\sigma_{g e o}$ is the constant contribution from geometrical imperfections of the detector estimated by the MC to be $14 \mu \mathrm{m}$. Based on this formula the measured half resolution of $39 \mu \mathrm{m}$ indicates a full detector resolution of about $23 \mu \mathrm{m}$. Several systematic effects were studied, e.g. alternative methods to obtain $\sigma_{g e o}$ and different ranges for the Gaussian fit, and from these a total error of $\pm 2 \mu \mathrm{m}$ was obtained.

\subsubsection{ALFA versus Si-telescope}

A dedicated reference tracking detector was not available during the regular ALFA testbeam period. A few runs could, however, be taken with track information from a high precision telescope, situated about $1 \mathrm{~m}$ upstream of the ALFA setup, with which the DEPFET collaboration performed measurements. The data acquisition systems of the two experiments were synchronized during these runs. The tracks reconstructed by the telescope were extrapolated to the z-position of ALFA 
and after a software alignment of the telescope with the ALFA coordinate system, the track positions of both systems could be directly compared. Two scenarios were considered. In the first, the reconstruction efficiency was maximized by using loose cuts. In the second scenario, tighter cuts were applied on the number of layers with more than one hit and on the total amount of hits. With these quality cuts the resolution was optimized.

\begin{tabular}{|c|c|c|}
\hline & reconstruction cuts & quality cuts \\
\hline$\sigma_{H}[\mu \mathrm{m}]$ & $56 \pm 3$ & $51 \pm 2$ \\
\hline$\sigma_{T}[\mu \mathrm{m}]$ & $46 \pm 2$ in $x$ and $66-77 \pm 4$ in $y$ \\
\hline$\sigma_{A L F A}[\mu \mathrm{m}]$ & $26 \pm 3$ & $25 \pm 3$ \\
\hline Efficiency [\%] & $96 \pm 3$ & $56 \pm 3$ \\
\hline
\end{tabular}

Table 1. Resolution (averaged over $x$ and $y$ ) and efficiency.

The residuals from the half-detectors and the telescope were studied in a first stage and from knowing $\sigma_{H}$, the resolution of the telescope, $\sigma_{T}$, at the position of ALFA was determined. Due to the $1 \mathrm{~m}$ distance between the telescope and ALFA, the intrinsic resolution of about $10 \mu \mathrm{m}$ inside the telescope was degraded to an observed telescope resolution at the ALFA position of about $46 \mu \mathrm{m}$ in $x$ and to $66-77 \mu \mathrm{m}$ in $y$. The performance of the telescope in the vertical direction varies with the hit region, which explains the observed variation of the resolution.

In a second stage, the spatial resolution of the full ALFA detector could be extracted. This was done by subtracting the calculated telescope contribution from the ALFA - telescope residual distribution. At the same time the ALFA efficiency was determined. The results are summarized in table 1 where it is shown that with basic reconstruction cuts a detection efficiency above $95 \%$ was obtained with a spatial resolution of about $26 \mu \mathrm{m}$. Both the stand-alone study and the study using the telescope showed that the resolution improves marginally for the full detector when quality cuts were applied while the efficiency drops drastically. The modest telescope resolution at the position of ALFA and the limited statistics implies relatively large errors, as seen in table 1, which were obtained from the spread of results in analyses using different runs.

\subsection{The overlap detectors}

The vertical distance between the two overlap detectors was reconstructed as the average difference between the positions of the fibres that were hit in each detector

$$
y_{O D R}=\frac{1}{n} \sum_{i=1}^{n}\left[\frac{1}{2}\left(y_{O D 2,1}+y_{O D 2,2}\right)-\frac{1}{2}\left(y_{O D 1,1}+y_{O D 1,2}\right)\right]
$$

where $y_{O D k, l}$ is the vertical position of the fibre hit in the layer $l$ of the detector $k$ and the sum is over the number of selected events (see figure \#). The events were selected on the basis of the number of fibres that were hit. Only events with a total of 4 hits and a single hit per plane were used for the reconstruction. The efficiency was only $11 \%$ due to the noise problems mentioned above. A good correlation was found between the reconstructed relative distance $y_{O D R}$ and the set value $y_{\text {set }}$ as shown in the left part of figure 5. The difference between $y_{O D R}$ and the straight line fit is in general smaller than $15 \mu \mathrm{m}$ (see right part of figure 5). Most of the positions were independently measured 


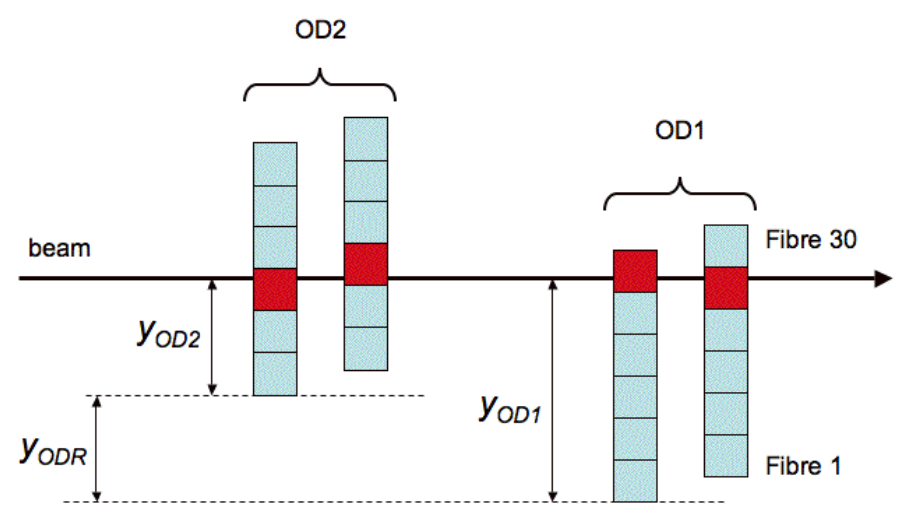

Figure 4. The vertical distance between the two overlap detectors is calculated from the measurement of particles which traverse both detectors: $y_{O D R}=y_{O D 2}-y_{O D 1}$.
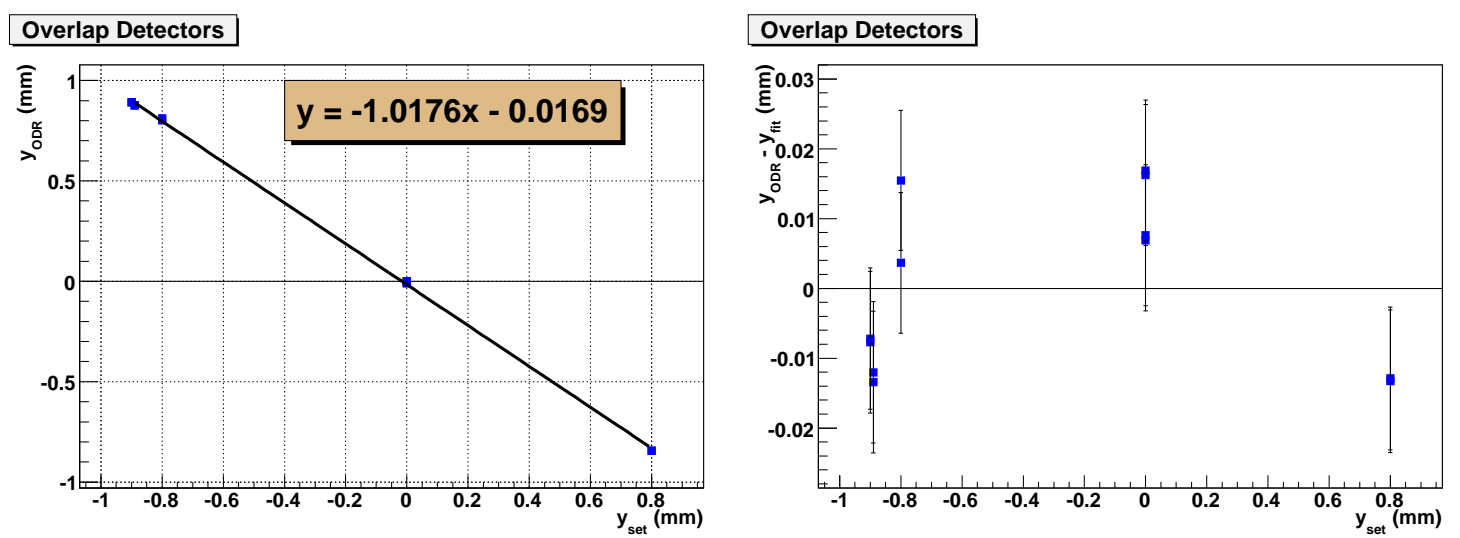

Figure 5. Reconstructed vertical distance $y_{O D R}$ between the two overlap detectors as a function of the mechanically set distance $y_{\text {set }}$ (left). The straight line is a fit to the data points. Difference between the reconstructed distance $y_{O D R}$ and the fit for the same data (right). The error bars represent the quadratic sum of the errors from the reconstruction algorithm and the errors of the mechanical measurement.

a second time. The two sets of reconstructed positions are compatible within the precision of the micrometric screw.

\section{Conclusions}

The ATLAS experiment is planning to measure the absolute luminosity from elastic scattering at very small angles using a scintillating fiber tracker called ALFA. The ALFA fiber detector was validated in a testbeam at DESY in November 2005, however, with standard electronics and using a $6 \mathrm{GeV} / \mathrm{c}$ electron beam.

A second testbeam was carried out at CERN using new prototypes together with the first version of the dedicated ALFA electronics, required for operation within ATLAS at the LHC, and 
using a high energy hadron beam. The high energy hadron beam approaches the final situation in the LHC and the spatial resolution was expected to improve with respect to the previous test because of reduced multiple scattering. A number of imperfections, likely to create fake noise hits, were identified during the tests of the electronics which will be corrected in the next version. An increased noise rate was also observed in the data with respect to the previous test where standard electronics was used, however, the tracking performance was still shown to be adequate for the purpose of the luminosity measurement. The space point reconstruction was studied by two different methods which showed resolutions of about $25 \mu \mathrm{m}$ and where a reconstruction efficiency above $95 \%$ was obtained.

Overlap detectors will be used for a precise vertical alignment of ALFA at the LHC. These detectors, as well as the alignment method, were tested for the first time in the CERN testbeam and it was shown that the required precision could be achieved, since the vertical relative alignment could be controlled to a precision better than $15 \mu \mathrm{m}$.

The natural next step will be to construct a full-size detector consisting of 10 planes with $2 \mathrm{x}$ 64 fibres. This full-size detector will be integrated in one Roman Pot unit and together with the overlap detectors and the trigger counters make a full system. We would also include all the frontend electronics together with the mother board. Such a complete system will allow us to evaluate and eliminate possible remaining electronics noise sources. Moreover the integration in the Roman Pot will permit us to address mounting and alignment precision in the Roman Pot and also relative alignment precision between the main detectors and the overlap detectors.

\section{Acknowledgments}

We would like to thank our technical staff for their competent work in building the fibre detectors and preparing the testbeam setup: A. Folley, L. Kottelat, J. Mulon, M. v. Stenis (all CERN), M. Szauter (University of Giessen) and J. Patriarca (LIP). We are grateful to the ALICE Silicon Pixel team, M. Burns and P. Riedler, for providing us with a motorized XYR table. We wish to acknowledge the work of the ATLAS TDAQ community in providing the underlying online software and infrastructure for read-out and dataflow. We would like to express our gratitude to M. Mathes and the DEPFET collaboration for allowing us to take common runs with their Silicon beam telescope and for providing us with the tracking data. We would like to acknowledge the effort of the SPS team for delivering stable beams and their help with the beam setup and tuning. Acknowledgments also to FCT/MCTES for grants and funding of the project "Collaboration in the ATLAS Experiment" POCI/FP/63936/2005.

\section{References}

[1] ATLAS Collaboration, ATLAS Forward Detectors for Measurement of Elastic Scattering and Luminosity Determination, ATLAS TDR, in preparation; ATLAS Collaboration, ATLAS Forward Detectors for Luminosity Measurement and Monitoring, CERN-LHCC/04-10, LHCC I-014.

[2] S. Ask et al., Luminosity measurement at ATLAS - development, construction and test of scintillating fibre prototype detectors, Nucl. Instr. and Meth. A568 (2006) 588-600. 
[3] V. Vorobel and H. Stenzel, GEANT4 simulation of the testbeam set-up for the ALFA detector, ATL-LUM-PUB-2006-006.

[4] A. Braem, C. Joram, A. Mapelli, A. Kocnar and J. Pospichal, Metrology results of the ALFA 2006 prototype detectors, ATL-LUM-PUB-2006-007.

[5] J. Santos, J.G. Saraiva, Y. Nunes, A. Wemans, A. Maio, M.J.P. Maneira, Aluminization of Scintillating Fibers for the Luminosity Detector of ATLAS, ATL-LUM-PUB-2007-005;

L. Gurriana et al., Quality Control of scintillating fibres for the ALFA/ATLAS prototype, ATL-COM-LUM-2007-004 (in preparation);

J. Santos et al., Characterization of plastic optical fibres with pulsed LEDs, Proceedings of 10th International Symposium on Radiation Physics (Accepted by NIM for Publication).

[6] A. Mapelli et al., Design, Construction and Metrology of the Overlap Detectors for the ALFA System, ATL-LUM-PUB-2007-004.

[7] P. Barrillon et al., MAROC: Multi-Anode ReadOut Chip for MaPMTs, Proceeding of IEEE - 2006 Nuclear Science Symposium, San Diego, USA, 29 Oct to 2 Nov 2006.

[8] ATLAS Collaboration, First Level Trigger TDR, CERN-LHCC/98-14, ATLAS TDR 12, (1998); P. Gallno, The ATLAS read out data flow control module and the TTC VME interface production status, Proceedings of the 7th Workshop on Electronics for LHC Experiments, Stockholm, Sweden, 10 to 14 Sep 2001;

P. Gallno, Modules development for the TTC system, Proceedings of the 5th Workshop on Electronics for the LHC Experiments (LEB 99), Snowmass, Colorado, 20 to 24 Sep 1999;

S. Baron, TTC challenges and upgrade for the LHC, Proceedings of the 11th Workshop on Electronics for LHC and Future Experiments, Heidelberg, Germany, 12 to 16 Sep 2005.

[9] ATLAS Collaboration, High-Level Trigger Data Acquisition and Controls, CERN-LHCC/03-22, ATLAS TDR 16, (2003);

B. Hallgren, H. Boterenbrood, H.J. Burckhart and H. Kvedalen, The Embedded Local Monitor Board (ELMB) in the LHC Front-end I/O Control System, Proceeding of the 7th Workshop on Electronics for LHC Experiments, Stockholm, Sweden, 10 to 14 Sep 2001;

I. Alexandrov et al., Online software for the ATLAS test beam data acquisition system, IEEE Trans. Nucl. Sci. 51 (2004) 578.

[10] P. Moreira et al., A radiation tolerant gigabit serializer for LHC data transmission, Proceedings of the 7th Workshop on Electronics for LHC Experiments, Stockholm, Sweden, 10 to 14 Sep 2001.

[11] W. Iwanski, S. Haas and M. Joos, A PCI Interface with four 2-Gbit/s optical links, IEEE TNS Dec 2005, Vol.52 Number 6, pages 2861-2865, ISSN 0018-9499.

[12] Rene Brun and Fons Rademakers, ROOT - An Object Oriented Data Analysis Framework, Proceedings AIHENP'96 Workshop, Lausanne, Sep. 1996,Nucl. Instr. and Meth. A389 (1997) 81-86. See also http://root.cern.ch/.

[13] S. Ask, S. Hoffmann and H. Stenzel, Spatial Resolution Study of ALFA from the CERN Testbeam in 2006, ATL-LUM-PUB-2007-003.

[14] P. Hough, Method and Means for Recognizing Complex Patterns, U.S. Patent 3,069,654, 1962; R.O. Duda and P.E. Hart, Use of the Hough Transform to Detect Lines and Curves in Pictures, Comm.ACM, Vol. 15 (1972) 11-15.

[15] A. Mapelli, ALFA Overlap Detectors Prototype Study from the CERN Testbeam in 2006, ATL-COM-LUM-2007-003 (in preparation). 